\title{
Diversidade de Lepidoptera (Papilionoidea e Hesperioidea) em dois fragmentos de floresta no município de Frederico Westphalen, Rio Grande do Sul, Brasil
}

\author{
Ricardo Giovenardi ${ }^{1}$, Rocco Alfredo Di Mare ${ }^{2}$, Jonas Sponchiado ${ }^{1}$, Suelem H. Roani ${ }^{1}$, Fábio A. F. Jacomassa ${ }^{3}$, \\ Augusto B. Jung ${ }^{1} \&$ Mirian A. Porn ${ }^{1}$
}

\begin{abstract}
'Departamento de Biologia, Universidade Regional Integrada do Alto Uruguai e das Missões - URI, Campus de Frederico Westphalen, Frederico Westphalen- RS. ricardogiovenardi@gmail.com

${ }^{2}$ Laboratório de Biologia Evolutiva Camobi, Departamento de Biologia, Centro de Ciências Naturais e Exatas, Campus Universitário, Faixa de Camobi km 09, 97119-900, Santa Maria-RS.

3Programa de Pós-Graduação em Biologia, UNISINOS, São Leopoldo-RS.
\end{abstract}

\begin{abstract}
Diversity of Lepidoptera (Papilionoidea e Hesperioidea) in two fragments in Frederico Westphalen, Rio Grande do Sul State, Brazil. A systematic survey was carried out in two localities of the Municipal District of Frederico Westphalen, fragment of Villa Faguense and Schoenstatt's Sanctuary, situated 1,500 m apart, from March 2005 to April 2006. In 140 hours of sampling, 3123 individuals, distributed in 169 species, were registered. Nymphalidae was the most sampled with approximately $53 \%$ of the recorded species. Nine species sampled were still no registered for the State. The Shannon-Wiener diversity index was higher in fragment of Villa Faguense, place less disturbed. The Simpson and BergerParker dominance indexes had been more representative in fragment of Schoenstatt Sanctuary, with higher number of abundant species. Among the species captured $126(74,55 \%)$ are accidental, $25(14,80 \%)$ are accessory and $18(10,65 \%)$ are constant.
\end{abstract}

KEYWORDS. Butterfly conservation; Lepidoptera ecology; species richness.

\begin{abstract}
RESUMO. Diversidade de Lepidoptera (Papilionoidea e Hesperioidea) em dois fragmentos de floresta no município de Frederico Westphalen, Rio Grande do Sul, Brasil. Foram realizados levantamentos mensais em duas localidades no Município, fragmento da Vila Faguense e do Santuário de Schoenstatt, os quais distam entre si em $1.500 \mathrm{~m}$, entre março de 2005 e abril de 2006. Em 140 horas de amostragem foram registrados 3.123 indivíduos distribuídos em 169 espécies. Nymphalidae foi a família mais amostrada com aproximadamente 53\% das espécies registradas. Nove espécies ainda não haviam sido registradas para o Estado. O índice de Diversidade de Shannon-Wiener foi maior no fragmento da Vila Faguense, local menos perturbado. Os índices de dominância de Simpson e Berger-Parker foram mais representativos no Santuário de Schoenstatt, com maior número de espécies abundantes. Foram registradas em Frederico Westphalen 126 (74,55\%) espécies acidentais, 25 acessórias $(14,80 \%)$ e 18 constantes $(10,65 \%)$.
\end{abstract}

PALAVRAS-CHAVE. Conservação de borboletas; ecologia; riqueza de espécies.

As borboletas estão representadas na região Neotropical com 7.784 espécies (Lamas 2004), e no Brasil com cerca de 3.200 espécies. Em função da sua importância nas pesquisas sobre biogeografia e interação inseto/planta podem ser utilizadas como bioindicadores (Uehara-Prado et al. 2004), determinação de prioridades, planejamento e na administração de reservas naturais. Também são usadas em estudos de ecologia de populações, dispersão e migração, genética da seleção natural e em fatores e processos básicos, como alimentação, predação, parasitismo, competição e defesa (Brown Jr. 1992).

À medida que aumenta a pressão antrópica no planeta, aumentam as ameaças aos ecossistemas, principalmente os tropicais (Wood \& Gillman 1998). As florestas estão sendo reduzidas a poucas áreas, cada vez mais degradadas e convertidas ao agroecossistema (Daily \& Ehrlich 1995) e o monitoramento de populações e comunidades de borboletas ao longo do tempo pode fornecer informações importantes para que medidas sejam tomadas antes que os efeitos da perturbação ambiental sejam irreversíveis (Uehara-Prado et al. 2004).
No Estado do Rio Grande do Sul, alguns dos trabalhos realizados não apresentam informações a respeito do esforço de coleta utilizado e dos locais estudados. Este trabalho pretende caracterizar a fauna de borboletas ocorrentes em remanescentes da Floresta Estacional Decidual do norte do Rio Grande do Sul, em Frederico Westphalen, através da listagem, medidas da riqueza, abundância, diversidade, dominância e constância de espécies, com a finalidade de contribuir para o conhecimento da lepidopterofauna da região.

\section{MATERIALE MÉTODOS}

Caracterização das áreas estudadas. De acordo com informações da Fundação SOS Mata Atlântica (2006), a região do Alto Uruguai, onde está localizado o Município de Frederico Westphalen, estava 100\% coberta de Floresta Atlântica latu senso, classificada em Floresta Estacional Decidual e Floresta Ombrófila Mista. Segundo Rambo (1961), as florestas estacionais são as formações com maior área de cobertura entre as ocorrentes no estado, predominando no Alto Uruguai, 
ao longo das encostas da Serra Geral e leste do Planalto SulRiograndense ou Serra do Sudeste. Através do Alto Uruguai, estende-se uma densa floresta, caracterizada por um estrato arbóreo superior, formado por árvores altas e emergentes, na sua maioria decidual (Reitz et al. 1988).

Localizado no planalto meridional, norte do Rio Grande do Sul (RS), o Município de Frederico Westphalen $\left(27^{\circ} 21^{\prime} \mathrm{S}\right.$ e $53^{\circ} 23^{\prime} \mathrm{W}$ ) possui relevo de patamares estruturais, com topografia suavemente ondulada, com altitudes entre 300 a 600 metros. Sua rede hidrográfica pertence à bacia do Rio Uruguai e o clima é descrito como subtropical úmido (Cfa), com chuva bem distribuída durante o ano e temperatura média do mês mais quente superior a $22^{\circ} \mathrm{C}$ (IBGE, 1985). Frentes frias podem ocasionar bruscas mudanças de temperatura em qualquer época do ano. Dados provenientes do Laboratório de Agrometeorologia da URI-FW, Estação Meteorológica da Universidade Regional Integrada do Alto Uruguai e das Missões revelam que a temperatura média anual é $18^{\circ} \mathrm{C}$, com valores mínimos entre $6^{\circ} \mathrm{C} \mathrm{e} 10^{\circ} \mathrm{C}$ no inverno e $31^{\circ} \mathrm{C}$ no verão. A precipitação média anual oscila entre $1.800 \mathrm{~mm}$ e $2.100 \mathrm{~mm}$, bem distribuída ao longo do ano. As chuvas são suficientes para as necessidades ambientais de água, com um volume de $800 \mathrm{~mm}$ a $900 \mathrm{~mm}$ de água excedente e disponível para o escoamento superficial (IBGE 1985).

Foram estudados dois fragmentos de mata, da Vila Faguense e do Santuário de Schoenstatt, com cerca de 35 ha e 50 ha de mata, respectivamente, os quais são característicos da região, formados por uma vegetação nativa perturbada pela antropização. A distância entre os fragmentos é aproximadamente 1.500 metros sem conexão entre as áreas.

$\mathrm{O}$ fragmento da Vila Faguense é o menos perturbado embora seja circundado por lavouras de diversos cultivos. Por toda a extensão do fragmento cruza o Rio Lajeado Tunas, diferenciando do outro estrato de mata estudado.

$\mathrm{O}$ fragmento de Schoenstatt, geralmente, freqüentado por pessoas nos fins de semana, é também circundado por lavouras, apresentando uma clareira em seu centro, ocupada para o cultivo da soja.

Foram utilizadas trilhas preexistentes no interior da mata para realização do trabalho.

Coleta dos dados. As coletas foram realizadas mensalmente, de março de 2005 a abril de 2006, entre as $09 \mathrm{~h} 00$ e $14 \mathrm{~h} 00$, percorrendo a borda e o interior da mata. O esforço foi de cerca de 5 horas-rede/local, ao longo das trilhas percorridas uma única vez em cada amostragem, com base na metodologia proposta por Pollard (1977).

As borboletas foram capturadas com rede entomológica, registradas em caderneta de campo ou acondicionadas em envelopes entomológicos e levadas ao Museu Regional do Alto Uruguai (MuRAU) da URI-FW, caso não fosse possível a identificação em campo. Foi capturado um exemplar de cada espécie para montagem e depositadas no Laboratório de Biologia Evolutiva da Universidade Federal de Santa Maria (UFSM).

A identificação das espécies foi feita com auxílio de bibliografia especializada (Ackery \& Vane-Wright 1984; Ackery 1988; Blandin 1988; Blandin 1993; Brown Jr. 1992; Canals 2003; Casagrande 1995; D’Abrera 1981, 1987a, b, c, 1988, 1994, 1995;
Le Moult \& Real 1962, 1963; Mielke 1994; Otero 1986). A nomenclatura utilizada seguiu Lamas (2004) e Mielke (2005).

Análise dos dados. Para a confirmação dos novos registros foram consultados os diversos inventários de borboletas realizados no Rio Grande do Sul.

As espécies representadas por um único indivíduo foram denominadas "singletons" (Novotny \& Basset 2000). Foram estimados os índices de Constância, de Diversidade de Shannon-Wiener (H'), índices de Dominância de Berger-Parker (d), Simpson (D) e o teste do Qui-quadrado. A análise dos dados e as estimativas dos índices foram realizadas utilizando o programa DivEs v2.0 (Rodrigues 2005) e para a curva do coletor utilizou-se Statistica for Windows, versão 7.0 (Statsoft 1995).

Para discussão e comparação com outros levantamentos a família Riodinidae foi tratada como subfamília de Lycaenidae conforme Iserhard \& Romanowski (2004) e Dessuy \& Moraes (2007).

\section{RESULTADOSEDISCUSSÃO}

Riqueza e abundância das espécies. Em 140 horas-rede, foram registrados 3.123 indivíduos, de 169 espécies, 6 famílias e 21 subfamílias. Do total de indivíduos, 2.423 (77,58\%) pertencem a Nymphalidae; 175 (5,60\%) a Papilionidae; 316 $(10,12 \%)$ a Pieridae; $168(5,38 \%)$ a Hesperiidae, $23(0,74 \%)$ a Lycaenidae e $18(0,58 \%)$ a Riodinidae (Tab. I).

Entre as espécies registradas, 93 pertencem à Nymphalidae (53\%), 34 Hesperiidae (21\%), 11 Papilionidae (7\%), 14 Pieridae (8\%), 11 Lycaenidae (7\%) e 6 Riodinidae (4\%). O número de espécies manteve-se sempre crescente ao longo do período de amostragem. A figura 1 mostra as equações e curvas logarítmicas ajustadas da curva do coletor obtidas para o fragmento da Vila Faguense (y = 47,0979 + 64,4975 $\log (\mathrm{x})$ ) e para o fragmento do Santuário de Schöentatt (y =7,257+ $81,2037 \log (\mathrm{x}))$. O aumento cumulativo médio de espécies por dia de captura foi de 4,31\% no fragmento da Vila Faguense e $5,15 \%$ no fragmento do Santuário de Schoentatt. Nas duas localidades o esforço amostral empreendido não foi suficiente para alcançar um patamar estável.

Uma comparação da riqueza de espécies por família observadas entre registros para o Brasil (Brown Jr. \& Freitas 1999) (sem identificação do esforço), para o Vale do Rio Maquiné (RS) (Iserhard \& Romanowski 2004) (238 horas-rede), Parque Estadual de Itapuã (RS) (Marchiori \& Romanowski 2006) (108 horas-rede) e o presente trabalho (140 horas-rede) mostra valores diferentes em relação ao Brasil e semelhantes ao Rio Grande do Sul (Tabela II). Emery et al. (2006), para Papilionoidea, no Distrito Federal, obteve 19 subfamílias, valor semelhante ao presente trabalho com 21 subfamílias, sendo 19 de Papilionoidea e 2 de Hesperioidea (Fig. 2), devido ao esforço amostral empregado. Ainda há de se destacar as diferenças das condições geográficas, já que o Rio Grande do Sul possui um clima subtropical característico entre as estações do ano, com inverno e verão bem diferenciados, o que pode afetar a fenologia das plantas e a sobrevivência de algumas espécies de borboletas (Iserhard \& Romanowski 2004). Devido a estas diferenças, Mielke \& Casagrande (1977) enfatizam a 
necessidade de ampliar o estudo de inventários de borboletas no sul do país.

Foram registradas 34 espécies de Hesperiidae de duas subfamílias, Pyrginae e Hesperiinae. Consideradas de difícil amostragem e identificação, são importantes como indicadoras de qualidade ambiental (Brown \& Freitas 1999). Para o estado, Biezanko \& Mielke (1973) e Mielke (1979/1980a, b) registraram 310 espécies de Hesperiidae, não especificando o esforço amostral.

Conforme Brown Jr. \& Freitas (1999), os ninfalídeos são muito diversificados em hábitos e morfologia. Quadros et al. (2004), em um período de dois anos, registraram 323 exemplares de ninfalídeos de 97 espécies. Teston \& Corseuil (2001) e Biezanko (1960a, b, c, d) listaram 118 espécies de Nymphalidae para o Estado, dentre elas 19 espécies de Ithomiinae, enquanto neste estudo registraram-se seis espécies. Segundo Beccaloni \& Gaston (1995), estas espécies são bons indicadores ambientais, pois são bem conhecidas, é um grupo relativamente pequeno e de fácil amostragem.

A subfamília com maior riqueza de espécies foi Pyrginae (25). Entre as espécies mais abundantes, destaca-se Fosterinaria quantius (Godart, 1824) (116), no fragmento da Vila Faguense e Vanessa braziliensis (Moore, 1883) (146), no fragmento do Santuário de Schoesntatt.

De acordo com Brown Jr. \& Freitas (1999), Lycaenidae é quase tão diversificada quanto Nymphalidae e importante indicadora ambiental. Na Serra do Japi (SP), de 1981 a 1984, Brown Jr. (1992) registrou 158 espécies de Lycaenidae. Biezanko et al. (1978) registraram 49 espécies de Riodinidae no Rio Grande do Sul em mais de 30 anos de coletas, sendo somente Barbicornis basilis mona Westwood, 1851 comum com as observadas neste trabalho.

Muitos Papilionidae são indicadores de matas bem conservadas e de abundância de recursos hídricos (Brown Jr. \& Freitas 1999). Em trabalhos realizados no Rio Grande do Sul (Iserhard \& Romanowski 2004; Link et al. 1977; Schwartz \& Di Mare 2001; Teston \& Corseuil 1998) foram registradas 31 espécies. Destas, 11 foram coletadas em Frederico Westphalen, inclusive Pterourus scamander (Boisduval, 1836), considerada escassa na região Missioneira por Biezanko (1959a).

Os adultos de Pieridae alimentam-se em flores, com os

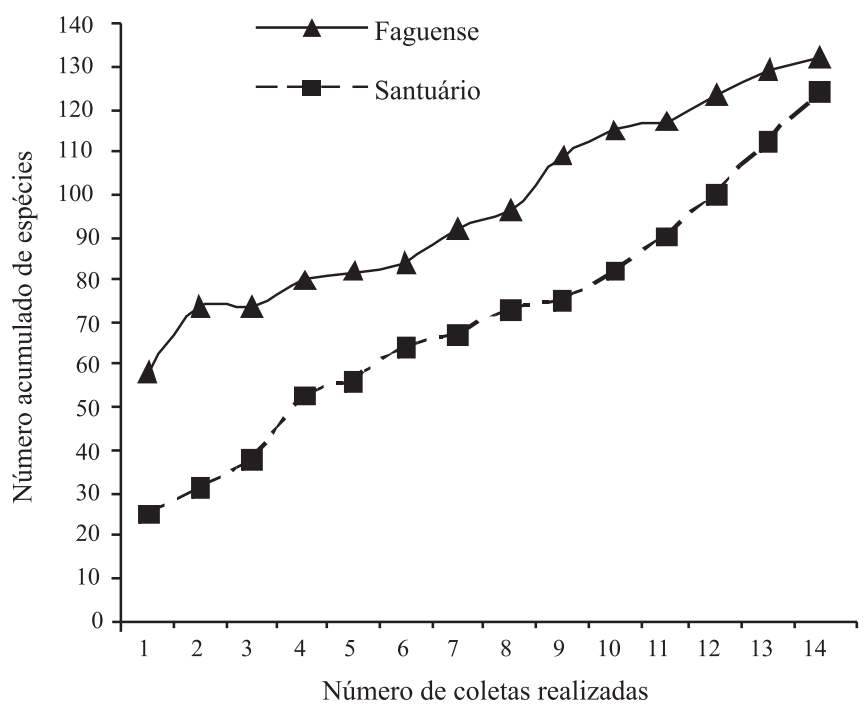

Fig. 1. Curva do coletor para o fragmento da Vila Faguense e do Santuário de Schoenstatt, Rio Grande do Sul de março de 2005 a abril de 2006.

machos pousando em areia úmida, em grandes bandos, sendo algumas espécies pragas e muitas migratórias (Brown Jr. 1992). Segundo Brown Jr. \& Freitas (1999) muitas espécies desta família podem ser indicadoras de perturbação no ecossistema. Das 38 espécies registradas no Estado (Iserhard \& Romanowski 2004; Link et. al 1977), 8 foram observadas no presente estudo. Destaca-se Ascia monuste orseis (Godart, 1819) espécie de interesse econômico (Teston \& Corseuil 2000).

Baz \& Boyero (1995) sugerem que o número de espécies de lepidópteros aumenta quando o fragmento é menos isolado de outros fragmentos, fator ocorrente para Frederico Westphalen, pois os locais de estudo são circundados por matas vizinhas. Para 9 das espécies ocorrentes não foram encontrados registros para o Estado.

Diversidade, dominância e constância das espécies. $O$ maior valor para o índice de Diversidade de Shannon-Wiener foi registrado no fragmento da Vila Faguense $(1,754)$, local com maior riqueza de espécies e menor abundância relativa em

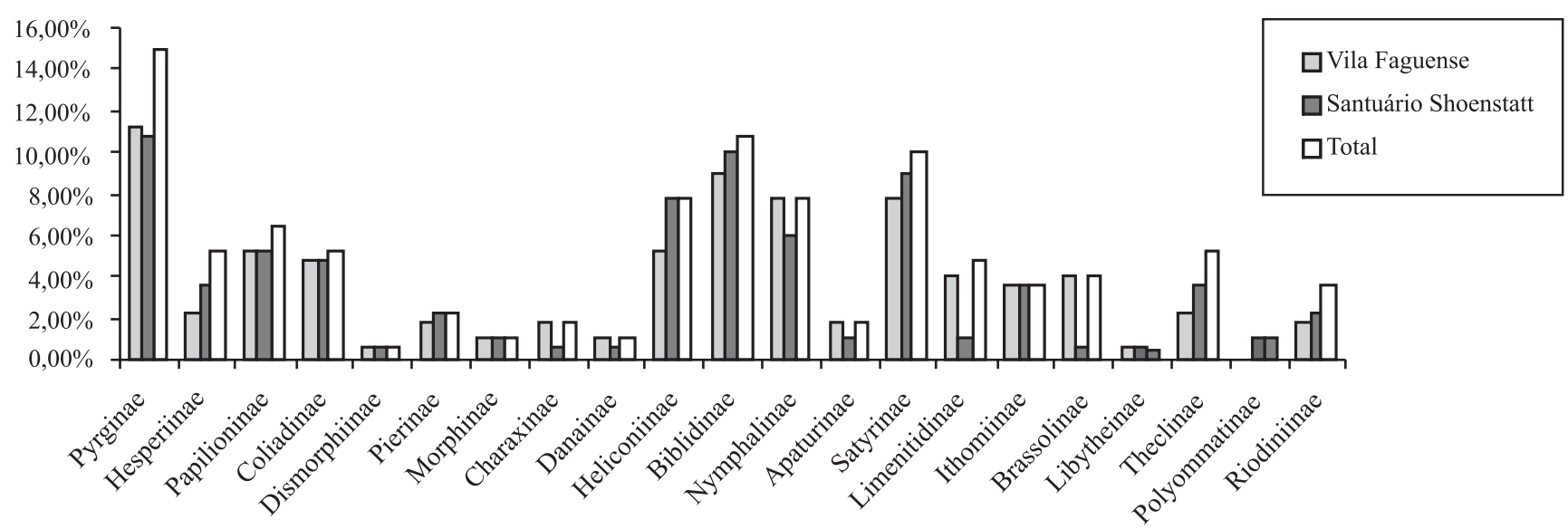

Fig. 2. Distribuição de Lepidoptera, por subfamília, nos fragmentos do Santuário de Schoenstatt e Vila Faguense, Rio Grande do Sul. 
Tabela I. Distribuição e abundância relativa de borboletas por famílias, subfamílias e espécies registradas (N) nos fragmentos da Vila Faguense e Santuário de Schoenstatt, em Frederico Westphalen, Rio Grande do Sul, de março de 2005 a abril de 2006, incluindo espécies representadas por um único indivíduo (singletons*) e novos registros de espécies para o Rio Grande do Sul (\#)

Táxon Faguense Santuário Total

\section{Hesperiidae $(\mathrm{N}=34)$}

Pyrginae $(\mathbf{N}=25)$

Achylodes busirus rioja Evans, 1953

Aethilla echina coracina Butler, 1870*

Astraptes anaphus anaphus (Cramer, 1777)

Astraptes creteus siges (Mabille, 1903)

Astraptes enotrus (Stoll, 1781)*

Autochton zarex (Hübner, 1818)*

Carrhenes canescens Röber, 1925*

Celaenorrhinus similis Hayward, 1933

Erynnnis (Erynnides) funeralis

(Scudder \& Burgess, 1870)*

Heliopetes alana (Reakirt, 1868)

Heliopetes arsalte (Linné, 1758)

Heliopetes omrina (Butler, 1870)*

Nisoniades bipuncta (Schaus, 1902)

Phanus australis Miller, 1965*

Phocides polybius phanias (Burmeister, 1879)

Polygonus leo leo (Gmelin, [1790])

Pyrgus oileus (Linnaeus, 1767)

Pythonides lancea (Hewitson, 1868)

Urbanus albimargo rica Evans, 1952*

Urbanus dorantes (Stoll, 1790)

Urbanus doryssus albicuspis

(Herrich-Schäffer, 1869)\#

Urbanus proteus proteus (Linnaeus, 1758)

Urbanus simplicius (Stoll, 1790)

Urbanus teleus (Hübner, 1821)

Zera hyacinthinus servius (Plotz, 1884)*

Hesperiinae $(\mathbf{N}=9)$

Carystoides sicania sicania (Hewitson, 1786)*

Lento krexoides (Hayward, 1940)

Lycas argentea (Hewitson, 1866)*

Lychnuchoides ozias ozias (Hewitson, 1878)

Nyctelius nyctelius (Latreille, 1824)

Quinta cannae (Herrich - Schaffer, 1869)*

Synapte silius (Latreille, [1824])*

Vettius diversa diversa (Herrich-Schäffer, 1869)*

Zariaspes mys (Hübner, 1808)

Total de indivíduos 84

Papilionidae $(\mathrm{N}=11)$

\section{Papilioninae $(\mathrm{N}=11)$}

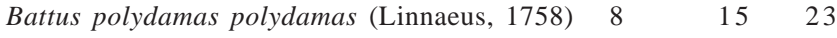

$\begin{array}{llll}\text { Battus polystictus polystictus (Butler, 1874) } & 2 & 20 & 22\end{array}$

Heraclides anchisiades capys (Hübner, 1809)* 1

Heraclides astyalus astyalus (Godart, 1819) 12

Heraclides hectorides (Esper, 1794) 15

Heraclides thoas brasiliensis

(Rothschild \& Jordan, 1906)

Mimoides lysithous rurik (Eschscholtz, 1821)

Parides agavus (Drury, 1782)

Parides anchises nephalion (Godart, 1819)

Protesilaus helios (Rothschilde \& Jordan, 1906)

Pterourus scamander scamander

(Boisduval, 1836)*

Total de indivíduos 52

Pieridae $(\mathrm{N}=14)$

Coliadinae $(\mathrm{N}=9)$

Anteos menippe (Hubner, [1818])

Eurema elathea flavescens (Chavannes, 1850)* 0

Eurema deva deva (Doubleday, 1847)
Tabela I. Continuação.

\begin{tabular}{lccc}
\hline Táxon & Faguense Santuário Total \\
\hline Aphrissa statira statira (Cramer, 1777) & 4 & 1 & 5 \\
Phoebis argante argante (Fabricius, 1775) & 7 & 10 & 17 \\
Phoebis neocypris neocypris (Hübner, 1823) & 35 & 47 & 82 \\
Phoebis philea philea (Linnaeus, 1763) & 2 & 0 & 2 \\
Phoebis sennae marcellina (Cramer, 1777) & 8 & 31 & 39 \\
Rhabdodryas trite banski (Breyer, 1939) & 4 & 47 & 51 \\
$\begin{array}{l}\text { Dismorphiinae (N=1) } \\
\text { Pseudopieris nehemia (Boisduval, 1836) }\end{array}$ & 3 & 3 & 6 \\
Pierinae (N=4) & & & \\
Ascia monuste orseis (Godart, 1819) & 21 & 6 & 27 \\
Glutophrissa drusilla drusilla (Cramer, 1777) & 14 & 7 & 21 \\
Pereute swainsoni (Gray, 1832) & 5 & 3 & 8 \\
Tatochila autodice (Hübner, 1818) & 0 & 2 & 2 \\
$\quad$ Total de indivíduos & $\mathbf{1 4 2}$ & $\mathbf{1 7 4}$ & $\mathbf{3 1 6}$
\end{tabular}

Nymphalidae $(\mathrm{N}=93)$

Morphinae $(\mathbf{N}=2)$

$\begin{array}{lllll}\text { Morpho aega aega (Hübner, [1822]) } & 26 & 7 & 33\end{array}$

Morpho helenor achillides C. Felder e R.Felder, $\begin{array}{lll}39 & 30 \quad 69\end{array}$ 1867

Charaxinae $(\mathrm{N}=3)$

Archaeoprepona demophoom (Hübner, [1814])* 11001

Mempphis moruus stheno (Prittwitz, 1865) $74 \quad 4 \quad 11$

Zaretis itys (Cramer, 1777)*

Danainae $(\mathbf{N}=\mathbf{2})$

Danaus gilippus gilippus (Cramer, 1775)

Danaus erippus (Cramer, 1775)

Heliconiinae $(\mathrm{N}=13)$

Actinote carycina Jordan, 1913

Actinote discrepans d'Almeida, 1958*

Actinote melanisans Oberthür, 1917*

Agraulis vanillae maculosa (Stichel, [1908])

Dione juno juno (Cramer, 1779)

Dione moneta (Hübner, [1825])

Dryadula phaetusa (Linnaeus, 1758)

Dryas iulia alcionea (Cramer, 1779)

Eueides aliphera aliphera (Godart, 1819)

Euptoieta claudia claudia (Cramer, 1775)*

Euptoieta hegesia meridiana Stichel, 1938\#

Heliconius erato phylis (Fabricius, 1775)

Heliconius ethilla narcaea (Godart, 1819)

Biblidinae $(\mathbf{N}=\mathbf{1 8})$

Biblis hyperia nectanabis (Fruhstorfer, 1909)

Callicore pygas thamyras (Menétries, 1857)*

Callicore hydaspes (Drury, 1782)*

Catonephele numilia neogermanica

(Stichel, 1899)

Diaethria candrena (Godart, 1824)

Diaethria clymena meridionalis Bates, 1864

Dynamine athemon (Linnaeus, 1758)\#

Dynamine postverta (Cramer, 1779)

Dynamine myrrhina (Doubleday, 1849)

Dynamine tithia tithia (Hübner, [1823])

Epiphile hubneri Hewitson, 1861*

Eunica eburnea Fruhstorfer, 1907

Hamadryas epinome

(C. Felder \& R. Felder, 1867)

Hamadryas februa (Hübner, [1823])

Hamadryas fornax (Hübner, [1823])

Myscelia orsis (Drury, 1782)\#

Smyrna blomfildia blomfilidia (Fabricius, 1781)*

Temenis laothoe meridionalis Ebert, 1961

Nymphalinae $(\mathrm{N}=13)$

Anartia amathea roeselia (Eschscholtz, 1821) 31

Chlosyne lacinia saundersi Doubleday, $1847 \quad 88$

Eresia lansdorfi (Godart, 1819)

Hypanarthia lethe (Fabricius, 1793)
11

19

1

0

12

3

2

30

0

0

94

0

48

\section{1
1}

1

1
5
1

1
2

16

2

0

22

22
11

32
5

5
3

3
0

3

31
88
2

25

7
1
8
2
16

3

1
11
1

$\begin{array}{ll}0 & 11\end{array}$

$17 \quad 36$

12

11

$1 \quad 1$

$6 \quad 8$

$0 \quad 3$

$\begin{array}{ll}0 & 2\end{array}$

$37 \quad 67$

45

11

$36 \quad 45$

$90 \quad 184$

$15 \quad 15$

$\begin{array}{ll}76 & 124\end{array}$

$\begin{array}{ll}1 & 1 \\ 0 & 1\end{array}$

56

34

38

56

68

$26 \quad 42$

13

11

24

$3 \quad 14$

$15 \quad 47$

16

14

$\begin{array}{ll}1 & 1 \\ 2 & 5\end{array}$

$35 \quad 66$

$45 \quad 133$

$\begin{array}{lc}0 & 2 \\ 8 & 33\end{array}$
$20 \quad 32$ 
Tabela I. Continuação.

\begin{tabular}{|c|c|c|c|}
\hline \multirow{2}{*}{$\begin{array}{l}\text { Táxon } \\
\text { Hypanartia bella (Fabricius, 1793) }\end{array}$} & \multicolumn{3}{|c|}{ Faguense Santuário Total } \\
\hline & 3 & 5 & 8 \\
\hline Junonia evarete (Cramer, 1779) & 79 & 131 & 210 \\
\hline Ortilia dicoma (Hewitson, 1864) & 2 & 0 & 2 \\
\hline Ortilia ithra (Kirby, 1871) & 20 & 46 & 66 \\
\hline Ortilia orthia (Hewitson, 1856) & 2 & 1 & 3 \\
\hline Siproeta epaphus trayja (Hübner, [1823]) & 13 & 18 & 31 \\
\hline Tegosa claudina (Eschscholtz, 1821) & 41 & 49 & 90 \\
\hline Vanessa braziliensis (Moore, 1883) & 35 & 146 & 181 \\
\hline Vanessa myrinna (Doubleday, 1849)* & 1 & 0 & 1 \\
\hline \multicolumn{4}{|l|}{ Apaturinae $(\mathrm{N}=3)$} \\
\hline Doxocopa kallina (Staudinger, 1886) & 13 & 10 & 23 \\
\hline Doxocopa laurentia laurentia (Godart, 1824) & 42 & 4 & 46 \\
\hline \multicolumn{4}{|l|}{ Satyrinae $(\mathrm{N}=17)$} \\
\hline Capronnieria abretia (Capronnier, 1874) & 20 & 1 & 21 \\
\hline Eteona tisiphone (Boisduval, 1836) & 0 & 21 & 21 \\
\hline Forsterinaria necys (Godart, 1824) & 5 & 17 & 22 \\
\hline Forsterinaria quantius (Godart, 1824) & 116 & 39 & 155 \\
\hline Hermeuptychia hermes (Fabricius,1775) & 28 & 73 & 101 \\
\hline Manataria hercyna hercyna (Hübner, [1821]) & 2 & 0 & 2 \\
\hline Moneuptychia soter (Butler, 1877)* & 0 & 1 & 1 \\
\hline Moneuptychia paeon (Godart, [1824])* & 1 & 0 & 1 \\
\hline Pareuptychia summandosa (Gosse, 1880)\# & 4 & 3 & 7 \\
\hline Paryphthimoides phronius (Godart, [1824]) & 0 & 1 & 1 \\
\hline Paryphthimoides poltys (Prittwitz, 1865) & 36 & 4 & 40 \\
\hline Praepedaliodes phanias (Hewitson, 1862) & 6 & 27 & 33 \\
\hline Splendeuptychia libitina (Butler, 1870) & 1 & 1 & 2 \\
\hline Taygetis laches marginata Staudinger, [1887]\# & 1 & 2 & 3 \\
\hline Taygetis ypthima (Hübner, [1821]) & 8 & 1 & 9 \\
\hline Yphthimoides sp. Forster, 1964* & 0 & 1 & 1 \\
\hline Yphthimoides celmis (Godart, [1824])* & 1 & 26 & 27 \\
\hline \multicolumn{4}{|l|}{ Limenitidinae $(\mathrm{N}=8)$} \\
\hline Adelpha abia (Hewitson, 1850) & 1 & 0 & 1 \\
\hline Adelpha malea goyama Schaus, $1902 *$ & 1 & 0 & 1 \\
\hline Adelpha mincia Hall, 1938 & 10 & 5 & 15 \\
\hline Adelpha mythra (Godart, [1824]) & 2 & 0 & 2 \\
\hline Adelpha serpa (Boisduval,1836)* & 1 & 0 & 1 \\
\hline Adelpha syma (Godart, 1824) & 8 & 0 & 8 \\
\hline Adelpha zea (Hewitson, 1850)* & 1 & 0 & 1 \\
\hline Marpesia petreus petreus (Cramer, 1776) & 0 & 9 & 9 \\
\hline \multicolumn{4}{|l|}{ Ithomiinae $(\mathrm{N}=6)$} \\
\hline Dircena dero celtina Burmeister, 1878 & 15 & 12 & 27 \\
\hline Epityches eиротре (Geyer, 1832) & 18 & 57 & 75 \\
\hline Mechanitis lysimnia lysimnia (Fabricius, 1793). & . 13 & 7 & 20 \\
\hline Methona themisto (Hübner, 1818) & 2 & 7 & 9 \\
\hline $\begin{array}{l}\text { Placidina euryanassa } \\
\text { (C. Felder \& R. Felder, 1860) }\end{array}$ & 5 & 1 & 6 \\
\hline Pteronymia sylvo ( Geyer, 1832) & 32 & 18 & 50 \\
\hline \multicolumn{4}{|l|}{ Brassolinae $(\mathrm{N}=7)$} \\
\hline Brassolis astyra Godart, [1824]* & 1 & 0 & 1 \\
\hline Brassolis sophorae vulpeculus Stichel, 1902 & 2 & 0 & 2 \\
\hline Caligo brasiliensis Fruhstorfer, 1912*\# & 1 & 0 & 1 \\
\hline Dynastor darius darius (Fabricius, 1775)* & 1 & 0 & 1 \\
\hline Eryphanis reevesii Doubleday, 1849 & 8 & 2 & 10 \\
\hline Eryphanis reevesii Doubleday, 1849 & 8 & 2 & 10 \\
\hline Opsiphanes invirae amplificatus (Hübner, 1818) & 3 & 0 & 3 \\
\hline Opoptera aorsa (Godart, [1824])* & 1 & 0 & 1 \\
\hline \multicolumn{4}{|l|}{ Libytheinae $(\mathrm{N}=1)$} \\
\hline Libytheana carinenta carinenta Cramer, 1777 & 2 & 1 & 3 \\
\hline Total de indivíduos & s 1166 & 1257 & 2423 \\
\hline \multicolumn{4}{|l|}{ Lycaenidae $(\mathrm{N}=11)$} \\
\hline \multicolumn{4}{|l|}{ Theclinae $(\mathrm{N}=9)$} \\
\hline Arawacus separata (Lathy, 1926) & 5 & 2 & 7 \\
\hline Atlides polybes (Linnaeus, 1763)* & 1 & 0 & 1 \\
\hline Chalybs hassan (Stoll, 1790)* & 1 & 0 & 1 \\
\hline Laothus phydela (Hewitson, 1867)* & 0 & 1 & 1 \\
\hline
\end{tabular}

Tabela I. Continuação.

\begin{tabular}{lccc}
\hline Táxon & Faguense Santuário Total \\
\hline Ocaria thales (Fabricius, 1793)* & 0 & 1 & 1 \\
Parrhasius orgia (Hewitson, 1867) & 0 & 5 & 5 \\
Rekoa palegon (Cramer, 1780) & 0 & 3 & 3 \\
Symbiopsis strenua (Hewitson, 1877)* & 0 & 1 & 1 \\
Theritas curitibaensis (K. Johnson, 1992)* & 1 & 0 & 1 \\
Polyommatinae (N=2) & & & \\
Hemiargus hanno hanno (Stoll,1790)* & 0 & 1 & 1 \\
Leptotes cassius cassius (Cramer,1775)* & 0 & 1 & 1 \\
& $\mathbf{N 8}$ & $\mathbf{1 5}$ & $\mathbf{2 3}$ \\
Riodinidae (N=6) & & & \\
Riodiniinae (N=6) & & & \\
Barbicornis basilis mona Westwood, 1851* & 1 & 0 & 1 \\
Emesis russula Stichel, 1810 & 0 & 2 & 2 \\
Eurybia pergaea (Geyer, 1832)\# & 2 & 0 & 2 \\
Lemonias zygia zygia (Hübner, [1807])*\# & 0 & 1 & 1 \\
Melanis smithiae (Westwood, 1851) & 1 & 9 & 10 \\
Synargis calyce brennus (Stichel, 1910) & 0 & 2 & 2 \\
Total de indivíduos & $\mathbf{4}$ & $\mathbf{1 4}$ & $\mathbf{1 8}$ \\
Total de indivíduos & $\mathbf{1 4 5 6}$ & $\mathbf{1 6 6 7}$ & $\mathbf{3 1 2 3}$ \\
\hline
\end{tabular}

relação ao fragmento do Santuário de Schöenstatt $(1,6994)$ (Tabela III).

Wettsteihn \& Schmid (1999) sugerem que a abundância de espécies é afetada pela degradação do hábitat. Kocher \& Willians (2000), em estudo realizado na América do Norte (EUA, Sul do Canadá e em alguns locais do México), afirmaram que as paisagens mais perturbadas antropicamente podem prover condições para uma maior abundância de espécies. No fragmento do Santuário de Schöesntatt, local mais antrópico, obteve-se menor riqueza de espécie, mas maior abundância relativa.

O índice de dominância de Simpson é derivado de índices heterogêneos que medem os pesos das espécies abundantes mais comuns em uma comunidade (Dias 2004). Neste estudo o valor deste índice foi mais representativo no fragmento do Santuário de Schoenstatt $(0,0322)$ em relação ao fragmento da Vila Faguense $(0,028)$. Para a dominância de espécies foi empregado o índice de Berger-Parker, o maior valor também ocorreu no fragmento do Santuário de Schoenstatt $(0,0896)$ em relação ao fragmento da Vila Faguense $(0,0795)$ (Tabela III).

Analisando o número de espécies por localidade, 90 $(53,25 \%)$ foram comuns em ambos os locais. Das espécies registradas, $79(46,75 \%)$ ocorreram somente em um dos locais.

Tabela II. Comparação da riqueza de espécies por família (\%) de borboletas no Brasil e no Rio Grande do Sul. B \& F - Brown \& Freitas (1999), I \& R - Iserhard \& Romanowski (2004), M \& R - Marchiori \& Romanowski (2006), FW - Frederico Westphalen.

\begin{tabular}{lcccc}
\hline & BRASIL & \multicolumn{3}{c}{ RS } \\
\hline Nymphalidae & B\&F & I\&R & M\&R & FW \\
Pieridae & 24 & 36 & 57 & 53 \\
Papilionidae & 2 & 8 & 8 & 8 \\
Lycaenidae & 2 & 4 & 5 & 7 \\
Riodinidae & 36 & 19 & 5 & 7 \\
Hesperiidae & $\mathrm{X}$ & $\mathrm{X}$ & $\mathrm{X}$ & 4 \\
\hline
\end{tabular}


Tabela III. Número de espécies, número de indivíduos, índice de Diversidade de Shannon-Wiener, índice de Dominância de Simpson e de Berger-Parker, espécies registradas exclusivamente em um dos locais e número de espécies representadas por somente um indivíduo (Singleton), registradas nos fragmentos da Vila Faguense e Santuário de Schöenstatt, entre março de 2005 e abril de 2006.

\begin{tabular}{lccc}
\hline & Faguense & Santuário & Total \\
\hline Número de espécies & 133 & 128 & 169 \\
Número de indivíduos & 1456 & 1667 & 3123 \\
Shannon-Wiener(H') & 1,754 & 1,6994 & 1,7867 \\
Dominância de Simpson & 0,028 & 0,0322 & 0,0152 \\
Dominância de Berger-Parker & 0,0795 & 0,0896 & 0,0473 \\
Exclusivas & 42 & 37 & 79 \\
Singleton & 26 & 26 & 52 \\
\hline
\end{tabular}

No fragmento do Santuário de Schoenstatt 37 espécies $(21,89 \%)$ foram exclusivas e no fragmento da Vila Faguense 42 $(24,85 \%)$ (Tabela III).

Das espécies registradas em Frederico Westphalen, 52 $(30,77 \%)$ foram representadas por somente um indivíduo ("singletons") (Tab. III). Segundo Novotny \& Basset (2000), tais espécies podem ser transeuntes, que não estão no local para fins de alimentação; espécies, aparentemente raras, porque foram amostradas por ineficientes métodos; especialista e com baixo nível populacional; generalistas, alimentando-se ocasionalmente no local ou planta estudada ou especialista, alimentando-se na planta estudada. Muitas das espécies "raras" são muito difíceis de encontrar em qualquer lugar ou época, até mesmo quando presentes, uma vez que se mantêm em populações pequenas, sazonais e erráticas (Brown Jr. \& Freitas 1999). Os valores obtidos para as estimativas do Qui-quadrado para o fragmento da Vila Faguense $\left(\mathrm{X}_{(0,05 ; 21 \mathrm{GL})}^{2}=4,17\right)$ e para o fragmento do Santuário de Schöenstatt $\left(\mathrm{X}_{(0,05 ; 21 \mathrm{GL})}^{2}=1,09\right)$ mostram que as proporções de espécies obtidas nas subfamílias não diferem estatisticamente em cada uma das áreas analisadas isoladamente. Analisando em conjunto, as duas localidades não diferem quanto às proporções de espécies obtidas por subfamília, podendo ser reunidas como uma única amostra $\left(\mathrm{X}_{(0,05 ; 20 \mathrm{GL})}^{2}\right)=5,26$ (Tab. IV). Com base em Silveira Neto et al. (1976) foram registradas em Frederico Westphalen $126(74,55 \%)$ espécies acidentais, 25 (14,80\%) espécies acessórias e 18 $(10,65 \%)$ espécies constantes.

Agradecimentos. Os autores agradecem aos Drs. Alexandre Specht, Elio Corseuil, Olaf H. H. Mielke e Mirna Martins Casagrande pela correção do trabalho e a Márlon Paluch pelo auxílio na identificação das espécies e à Universidade Regional Integrada do Alto Uruguai e das Missões, Campus de Frederico Westphalen pela disponibilidade do MuRAL para triagem e identificação das borboletas.

\section{REFERÊNCIAS}

Ackery, P. R. 1988. Host plants and classification: a review of nymphalid butterflies. Biological Journal of the Linnaean Society 33: 95-203.

Ackery, P. R \& R. I. Vane-Wright. 1984. Milkweed butterflies their cladistics and biology. London: British Museum (Natural History), $425 \mathrm{p}$.
Tabela IV. Espécies observadas, esperadas e Qui-quadrado das subfamílias registrados nos fragmentos da Vila Faguense e Santuário de Schöenstatt, entre março de 2005 e abril de 2006.

\begin{tabular}{lccc}
\hline Subfamílias & $\begin{array}{c}\text { Observadas } \\
\text { Faguense/ } \\
\text { Santuário }\end{array}$ & $\begin{array}{c}\text { Esperadas } \\
\text { Faguense/ } \\
\text { Santuário }\end{array}$ & $\begin{array}{c}\text { Qui-quadrado } \\
\text { Faguense/ } \\
\text { Santuário }\end{array}$ \\
Pyrginae & $21 / 19$ & $21,40 / 20,15$ & $0,01 / 0,07$ \\
\hline Biblidinae & $14 / 17$ & $18,21 / 17,21$ & $0,97 / 0,00$ \\
Nymphalinae & $14 / 10$ & $15,50 / 10,71$ & $0,15 / 0,05$ \\
Satyrinae & $13 / 16$ & $13,19 / 14,69$ & $0,00 / 0,12$ \\
Heliconiinae & $9 / 11$ & $11,23 / 12,54$ & $0,05 / 0,17$ \\
Papilioniinae & $9 / 9$ & $9,56 / 9,14$ & $0,03 / 0,00$ \\
Coliadinae & $8 / 8$ & $8,13 / 7,81$ & $0,00 / 0,00$ \\
Ithomiinae & $7 / 6$ & $6,92 / 5,69$ & $0,00 / 0,02$ \\
Limenitidinae & $7 / 2$ & $5,89 / 1,88$ & $0,21 / 0,01$ \\
Morphinae & $5 / 3$ & $5,01 / 3,02$ & $0,00 / 0,00$ \\
Brassolinae & $4 / 0$ & $4,27 / 0,00$ & $0,02 / 0,00$ \\
Hesperiinae & $4 / 6$ & $3,63 / 4,86$ & $0,04 / 0,00$ \\
Theclinae & $4 / 6$ & $3,09 / 6,67$ & $0,27 / 0,07$ \\
Riodiniinae & $3 / 4$ & $2,63 / 4,15$ & $0,05 / 0,01$ \\
Apaturinae & $3 / 2$ & $2,24 / 2,20$ & $0,26 / 0,02$ \\
Charaxinae & $3 / 1$ & $1,91 / 1,17$ & $0,63 / 0,03$ \\
Pierinae & $3 / 4$ & $1,62 / 3,54$ & $1,17 / 0,06$ \\
Danainae & $2 / 1$ & $1,38 / 1,37$ & $0,28 / 0,10$ \\
Dismorphiinae & $1 / 1$ & $1,17 / 1,61$ & $0,03 / 0,23$ \\
Libytheinae & $1 / 1$ & $1,00 / 1,00$ & $0,00 / 0,00$ \\
Polyommatinae & $0 / 2$ & $0,00 / 2,58$ & $0,00 / 0,13$ \\
TOTAL & $\Sigma=134 / 126$ & $\Sigma=137,98 / 131,99$ & $\Sigma=4,17 / 1,09$ \\
\hline
\end{tabular}

Baz, A. \& A. G. Boyero. 1995. The effects of forest fragmentation on butterfly communities in central Spain. Journal of Biogeography 22: $129-140$.

Beccaloni, G. W. \& K. J. Gaston. 1995. Predicting species richness of Neotropical forest butterflies: Ithomiinae (Lepidoptera, Nymphalidae) as indicators. Biological Conservation 71: 7786.

Biezanko, C. M. 1959. Ia. Papilionidae da Zona Sueste do Rio Grande do Sul. Arquivos de Entomologia, Série A: 1-17.

Biezanko, C. M. 1960a. III. Danaidae et Ithomidae da Zona Sueste do Rio Grande do Sul. Arquivos de Entomologia, Série A: 1-6.

Biezanko, C. M. 1960b. III. Danaidae et Ithomidae da Zona Missioneira do Rio Grande do Sul. Arquivos de Entomologia, Série B: 1-6.

Biezanko, C. M. 1960c. IV. Satyridae, Morphidae et Brassolidae da Zona Sueste do Rio Grande do Sul. Arquivos de Entomologia, Série A: 1-13.

Biezanko, C. M. 1960d. IV. Satyridae, Morphidae et Brassolidae da Zona Missioneira do Rio Grande do Sul. Arquivos de Entomologia, Série B: $1-10$.

Biezanko, C. M \& O. H. H. Mielke. 1973. Contribuição ao estudo faunístico dos Hesperiidae americanos. IV Espécies do Rio Grande do Sul, Brasil, com notas taxonômicas e descrições de espécies novas (Lepidoptera ). Acta Biológica Paranaense 2: 51-102.

Biezanko, C. M.; O. H. H. Mielke \& A.Wedderhoff. 1978. Contribuição ao estudo faunístico dos Riodinidae do Rio Grande do Sul, Brasil (Lepidoptera). Acta Biológica Paranaense7: 7-22.

Blandin, P. 1988. The genus Morpho Lepidoptera Nymphalidae. Part I. The subgenera Iphimedeia and Schwartzia. Venette, Sciences Nat, 104 p.

Blandin, P. 1993. The genus Morpho Lepidoptera Nymphalidae. Part I. The subgenera Cytheritis, Balachowskyna and Cypritis. Venette, Sciences Nat, 104 p.

Brown Jr., K. S. 1992. Borboletas da Serra do Japi: diversidade, habitats, recursos alimentares e variação temporal, p.142-186. In: L.P.C. Morellato (Org.). História Natural da Serra do Japi: ecologia 
e preservação de uma área florestal no sudeste do Brasil. Campinas: Unicamp/FAPESP, $321 \mathrm{p}$.

Brown Jr., K. S \& A. V. L. Freitas. 1999. Lepidoptera, p. 227-243. In: Brandão, C.R.F. \& E.M. Cancello (Eds.). Biodiversidade do Estado de São Paulo, Brasil. Invertebrados terrestres. São Paulo, FAPESP, XVI+279 p.

Canals, G. R. 2003. Mariposas de Misiones. Buenos Aires, L.O.L.A., $492 \mathrm{p}$.

Casagrande, M.. M. 1995. Notas sistemáticas sobre Brassolinae. I. Tribos (Lepidoptera, Nymphalidae). Revista Brasileira de Zoologia 12: 671-699.

D’Abrera, B. 1981. Butterflies of the Neotropical region. Part I. Papilionidae \& Pieridae. Melbourne: Landsowne, 172 p.

D’Abrera, B. 1987a. Butterflies of the Neotropical region. Part II. Danaidae, Ithomiidae, Heliconiidae \& Morphidae. Victoria: Hill House, 174-384.

D'Abrera, B. 1987b. Butterflies of the Neotropical region. Part III. Brassolidae, Acraeidae \& Nymphalidae (partim). Victoria: Hill House, 386-525.

D'Abrera, B. 1987c. Butterflies of the Neotropical region. Part IV. Nymphalidae (partim). Victoria: Hill House, 528-678.

D'Abrera, B. 1988. Butterflies of the Neotropical region. Part V. Nymphalidae (Conc.), Satyridae. Victoria: Hill House, 680-877.

D'Abrera, B. 1994. Butterflies of the Neotropical region. Part VI Riodinidae. Victoria: Hill House, 880-1096.

D’Abrera, B. 1995. Butterflies of the Neotropical region. Part VII. Lycaenidae. Victoria: Hill House, 1098-1270.

Daily, G. C. \& P. R. Ehrlich. 1995. Preservation of biodiversity in small rainforest patches: rapid evaluations using butterfly trapping. Biodiversity and Conservation 4: 35-55.

Dessuy, M. B. \& A. B. B. Moraes. 2007. Diversidade de borboletas (Lepidoptera, Papilionoidea e Hesperioidea) em fragmentos de Floresta Estacional Decidual em Santa Maria, Rio Grande do Sul, Brasil. Revista Brasileira de Zoologia 24: 108-120.

Dias, S. C. 2004. Planejando estudos de diversidade e riqueza: uma abordagem para estudantes de graduação. Acta Scientiarum 26: 373-379.

Emery, E. O; K. S. Brown Jr. \& C. E. G. Pinheiro. 2006. As borboletas (Lepidoptera, Papilionoidea) do Distrito Federal, Brasil. Revista Brasileira de Entomologia 50: 85-92.

Fundação S.O.S Mata Atlântica/ Instituto Nacional de Pesquisas Espaciais / Instituto Sócio Ambiental - S.O.S Mata Atlântica/ INPE/ ISA. 2006. Evolução dos remanescentes florestais e ecossistemas associados no domínio de mata atlântica. Disponível em: $\langle$ http://www.sosmatatlantica.org.br/secao=atlas $>$. Acesso em $22 \mathrm{de}$ setembro de 2006.

IBGE - Instituto Brasileiro de Geografia e Estatística. 1985. Coleção de Monografias Municipais - Frederico Westphalen, RS. Nova série, $16 \mathrm{p}$.

Iserhard, C. A. A \& H. P. Romanowski. 2004. Lista de espécies de borboletas (Lepidoptera, Papilionoidea e Hesperioidea) da região do rio Maquiné, Rio Grande do Sul, Brasil. Revista Brasileira de Zoologia 21: 649-662.

Kocher, S. D. \& E. H. Willians. 2000. The diversity and abundance of North American butterflies vary with habitat disturbance and geography. Journal of Biogeography 27: 785-794.

Lamas, G. (ed.) 2004. Checklist: Part 4A. Hesperioidea - Papilionoidea. In: J. B. Heppner (Ed). Atlas of Neotropical Lepidoptera 5A. Gainesville, Association for Tropical Lepidoptera, XXXVI + 439p.

Le Moult, E. \& P. Real. 1962. Les Morpho d'Amerique du Sud et Centrale. Historique-Morphologie-Systématique. Paris: Éditions du Cabinet Entomologique Le Moult, 262 p.

Le Moult, E. \& P. Real. 1963. Les Morpho d'Amerique du Sud et Centrale. Tome II. Planches en coulers et en noir. Paris: Éditions du Cabinet Entomologique Le Moult, 104 p.

Link, D.; C. M. Biezanko; M. F. Tarragó \& S. Carvalho. 1977. Lepidoptera de Santa Maria e arredores. I: Papilionidae e Pieridae.
Revista do Centro de Ciências Rurais 7: 381-389.

Marchiori, M. O \& H. P. Romanowski. 2006. Species composition and diel variation of a butterfly taxocene (Lepidoptera, Papilionoidea and Hesperioidea) in a restinga forest at Itapuã State Park, Rio Grande do Sul, Brasil. Revista Brasileira de Zoologia 23: 443454.

Mielke, O. H. H. 1979/1980a. Contribuição ao estudo faunístico dos Hesperiidae Americanos. V Nota Suplementar - As espécies de Pyrrhopyginae e Pyrginae do Rio Grande do Sul, Brasil (Lepidoptera). Acta Biológica Paranaense 8-9: 7-17.

Mielke, O. H. H. 1979/1980b. Contribuição ao estudo faunístico dos Hesperiidae Americanos. VI Nota Suplementar - As espécies de Hesperiinae do Rio Grande do Sul, Brasil (Lepidoptera). Acta Biológica Paranaense 8-9: 127-172.

Mielke, C. G. C. 1994. Papilionoidea e Hesperoidea (Lepidoptera) de Curitiba e seus arredores, Paraná, Brasil, com notas taxonômicas sobre Hesperiidae. Revista Brasileira de Zoologia 11: 759_ 776.

Mielke, O. H. H. 2005. Catalogue of the American Hesperioidea: Hesperiidae (Lepidoptera). Volume 1. Complementary and supplmentary parts to the checklist of the Neotropical region. Hesperioidea: Hesperiidae: Pyrrhopyginae. Curitiba. Sociedade Brasileira de Zoologia. Vol. 1. 125 p.

Mielke, O. H. H. \& M. M. Casagrande. 1977. Papilionoidea e Hesperioidea (Lepidoptera) do Parque Estadual do Morro do Diabo, Teodoro Sampaio, São Paulo, Brasil. Revista Brasileira de Zoologia 14: 967-1001.

Novotny, V. \& Y. Basset. 2000. Rare species in communities of tropical insect herbivores: pondering the mistery of singletons. Oikos 89: $564-572$

Otero, L. 1986. Borboletas: Livro do naturalista. Rio de Janeiro: MEC/FAE, $112 \mathrm{p}$.

Pollard, E. 1977. A method for assessing changes in the abundance of butterflies. Biological Conservation 12: 115-134.

Quadros, F. C.; A. L. Dorneles \& E. Corseuil. 2004. Ninfalídeos (Lepidoptera, Nymphalidae), ocorrentes no Norte da Planície Costeira do Rio Grande do Sul, Brasil. Biociências 12: 147-164.

Rambo, B. 1961. Migration routes of the south Brazilian rain florest. Pesquisa botânica 12: 1-54.

Reitz, R.; R. M. Klein \& A. Reis. 1988. Projeto Madeira do Rio Grande do Sul. Porto Alegre, SUDESUL-GERS-IBDF, 525 p.

Rodrigues, W. C. 2005. DivES - Diversidade de espécies. Versão 2.0. Software e Guia do Usuário. Disponível em: <http:// www.ebras.vbweb.com.br> Acesso em: 17 de outubro de 2006.

Schwartz, G. \& R. A. Di Mare. 2001. Diversidade de quinze espécies de borboletas (Lepidoptera, Papilionidae) em sete comunidades de Santa Maria, RS. Ciência Rural 3: 49-55.

Silveira Neto, S.; O.Nakano; D. Barbin \& N. A Villa Nova. 1976. Manual de Ecologia de Insetos. São Paulo, Ceres, 419 p.

Statsoft. 1995. Statistica for Windowns (Computer program manual). Tulsa, OK, Stat Soft.

Teston, J. A. \& E. Corseuil. 1998. Lista documentada dos Papilionídeos (Lepidoptera, Papilionidae) do Rio Grande do Sul, Brasil. Biociências 6: 81-94.

Teston, J. A. \& E. Corseuil. 2000. Lista documentada dos Pierídeos (Lepidoptera, Pieridae) do Rio Grande do Sul, Brasil. Biociências 2: $115-132$.

Teston, J. A. \& E. Corseuil. 2001. Ninfalídeos (Lepidoptera, Nymphalidae) ocorrentes no Rio Grande do Sul, Brasil. Parte I. Danainae e Ithominae. Biociências 9: 51-61.

Uehara-Prado, M.; Freitas, A. V. L.; Francini, R .B. \& K. S. Brown Jr. 2004. Guia das Borboletas Frugívoras da Reserva Estadual do Morro Grande e Região de Caucaia do Alto, Cotia (São Paulo). Biota Neotropica 4: 1-9.

Wood, B. \& M. P. Gillman. 1998. The effects of disturbance on forest butterflies using two methods of sampling in Trinidad. Biodiversity and Conservation 7: 597-616. 